\title{
Interpolação dos parâmetros da equação de chuvas intensas com uso do inverso de potências da distância
}

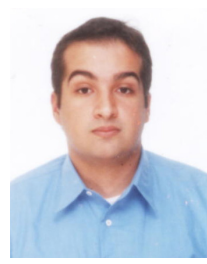

Roberto A. Cecílio \& Fernando F. Pruski²

\author{
1 DEA/UFV. CEP 36571-000, Viçosa, MG. Fone: (31) 3891-6739. E-mail: rcecilio@uai.com.br (Foto) \\ 2 DEA/UFV. E-mail: ffpruski@ufv.br
}

Protocolo 57 - 2/4/2003 - Aprovado em 31/10/2003

\begin{abstract}
Resumo: As equações de chuvas intensas representam excelente alternativa para a determinação das precipitações críticas utilizadas em projetos de engenharia; contudo, a sua determinação é muito trabalhosa. No Brasil, entretanto, dispõe-se, em diversos Estados, de um expressivo número de equações, o que torna possível a obtenção, por interpolação, dos parâmetros da equação de chuvas intensas para locais em que estes não são conhecidos. Neste trabalho compararam-se, considerando-se as informações disponíveis em 171 localidades do Estado de Minas Gerais, 625 diferentes combinações entre os quatro parâmetros da equação de chuvas intensas ("K", "a", "b" e "c") interpolados com a utilização da metodologia do inverso da potência da distância, através de cinco diferentes potências. Percebeu-se, em todas as combinações, tendência de superestimativa da intensidade de precipitação. A interpolação de "K" e "c" com o inverso da quinta potência da distância, "a" com o inverso da distância e "b" com o inverso do cubo da distância, apresentou melhores resultados na estimativa da intensidade de precipitação.
\end{abstract}

Palavras-chave: espacialização, dimensionamento hidráulico, escoamento superficial

\section{Interpolation of parameters of intensity-duration-frequency equation using the inverse distance to a different powers}

\begin{abstract}
Intense precipitation equations represent an excellent alternative to determinate critical rainfalls used in engineering designs but its obtainment is time-consuming. However, in some Brazilian States a great number of these equations have been determined for several places, permitting to obtain the equation parameters, by interpolation, for the places where these still have not bean known. In this paper 625 different combinations were compared for the four intense precipitation parameters ("K", "a", "b" and "c") interpolated by the inverse distance to a power method (using five different powers). Data from 171 places located in Minas Gerais State were used. In all the combinations, a tendency of overestimation was noticed for the precipitation intensity. "K" and "c" parameters interpolated by the inverse distance to the fifth power, "a" interpolated by the inverse distance of the first power and " $b$ " by the inverse distance to the third power showed better results in precipitation intensity prediction.
\end{abstract}

Key words: espatialization, hydraulic design, runoff

\section{INTRODUÇÃO}

O dimensionamento de drenos, vertedores de barragens e obras de proteção contra cheias e erosão hídrica, requer o estudo das precipitações intensas ocorridas no local de interesse, para a definição da chuva de projeto a partir da qual é definida a vazão a ser utilizada. Esta exigência geralmente é atendida a partir de informações pontuais, ou seja, são utilizadas equações do tipo intensidade-duração-freqüência (equações de chuvas intensas) derivadas de pluviógrafos específicos. Uma dificuldade freqüentemente enfrentada pelos técnicos é a inexistência dessa equação na localidade onde vai ser realizado o projeto, cujo tipo de problema pode ser contornado utilizandose a equação do pluviógrafo mais próximo, quando situado em região climática similar, ou interpolando resultados obtidos nas proximidades do local de interesse (Beltrame et al., 1991).

A determinação das equações de chuvas intensas apresenta, em muitos casos, grandes dificuldades em função da escassez de informações dessa natureza, da baixa densidade da rede de pluviógrafos e do pequeno período de observações disponível. Além disso a metodologia exige para sua obtenção, um exaustivo trabalho de tabulação, análise e interpretação de uma grande quantidade de pluviogramas. Por esta razão, ainda hoje poucos trabalhos têm sido desenvolvidos com tal finalidade, constituindo grande entrave na realização de projetos de obras hidráulicas mais confiáveis e econômicos (Pruski et al., 2002).

O Brasil dispõe de um número considerável de equações de chuvas intensas determinadas para diversas localidades 
dos Estados da Bahia, Espírito Santo, Minas Gerais, Paraná, Rio de Janeiro e São Paulo. Tais equações foram obtidas em estudos realizados por Fendrich \& Freitas (1992), Pinto et al. (1996), Silva et al. (1999a), Silva et al. (1999b), Freitas et al. (2001) e Silva et al. (2002).

Pruski et al. (2002) desenvolveram um "software" (PLÚVIO 1.3) que permite, utilizando-se procedimentos de interpolação, a determinação da equação de chuvas intensas para qualquer localidade dos Estados do Espírito Santo, Minas Gerais, Paraná, Rio de Janeiro e São Paulo. A interpolação é realizada independentemente para cada um dos parâmetros desta equação, sendo utilizadas todas as informações das localidades do Estado em que a equação é conhecida. O fator de ponderação utilizado corresponde ao inverso do quadrado da distância entre a localidade para a qual a equação é desejada e as localidades em que o parâmetro é conhecido.

Embora o uso de um fator de ponderação proporcional ao inverso do quadrado da distância tenha apresentado desempenho satisfatório no trabalho desenvolvido por Pruski et al. (1997), diversos fatores de ponderação têm sido também recomendados na literatura.

Tendo em vista a importância do conhecimento das equações de chuvas intensas, a dificuldade de sua determinação e a facilidade de interpolação dos parâmetros, quando se tem conhecimento de um número razoável de equações, o presente trabalho foi realizado com o objetivo de se comparar diferentes formas de interpolação dos parâmetros da equação de chuvas intensas, utilizando-se o método do inverso da potência da distância.

\section{MATERIAL E MÉTODOS}

A principal forma de caracterização de chuvas intensas é por meio da equação de intensidade, duração e freqüência da precipitação, representada por:

$$
i_{m}=\frac{K T^{a}}{(t+b)^{c}}
$$

em que:

$$
\begin{aligned}
& \mathrm{i}_{\mathrm{m}} \quad \text { - intensidade máxima média de precipitação, } \mathrm{mm} \mathrm{h}^{-1} \\
& \mathrm{~T} \quad \text { - período de retorno, anos } \\
& \mathrm{t}
\end{aligned}
$$

Utilizaram-se, como base de dados, os parâmetros das equações de chuvas intensas de 171 estações pluviográficas do Estado de Minas Gerais, determinados por Freitas et al. (2001).

Criou-se um mapa representativo da localização de cada estação pluviográfica, associando-se a cada uma os valores dos parâmetros da equação de chuvas intensas ("K", "a", "b", "c").

Com a utilização do "software" ArcView 3.2a procedeu-se à retirada de uma das 171 estações previamente escolhidas, realizando-se a interpolação de cada um dos quatro parâmetros da equação de chuvas intensas, através do método de interpolação do inverso de uma potência da distância. Neste procedimento de interpolação o valor atribuído à célula interpolada é obtido pela média ponderada que utiliza o peso dos postos de controle mais próximos (no caso, foram utilizadas todas as 170 estações restantes) ponderados pelo inverso da potência da distância, como mostra a Eq. 2.

$$
\mathrm{G}_{\mathrm{i}}=\frac{\sum_{\mathrm{i}=1}^{\mathrm{n}}\left(\frac{\mathrm{x}_{\mathrm{i}}}{\mathrm{d}_{\mathrm{i}}^{\mathrm{m}}}\right)}{\sum_{\mathrm{i}=1}^{\mathrm{n}}\left(\frac{1}{\mathrm{~d}_{\mathrm{i}}^{\mathrm{m}}}\right)}
$$

em que:

$\mathrm{G}_{\mathrm{i}}$ - Valor estimado na célula interpolada

$\mathrm{x}_{\mathrm{i}} \quad$ - Valor do i-ésimo posto de controle

$d_{i} \quad$ - distância euclidiana entre o i-ésimo posto de controle e a célula interpolada

m - expoente da distância euclidiana

$\mathrm{Na}$ interpolação dos parâmetros utilizaram-se cinco diferentes expoentes da distância: 1 (ID1), 2 (ID2), 3 (ID3), 4 (ID4) e 5 (ID5), totalizando 20 interpolações para cada estação. Este procedimento foi realizado separadamente para as 50 estações pluviográficas apresentadas na Figura 1.

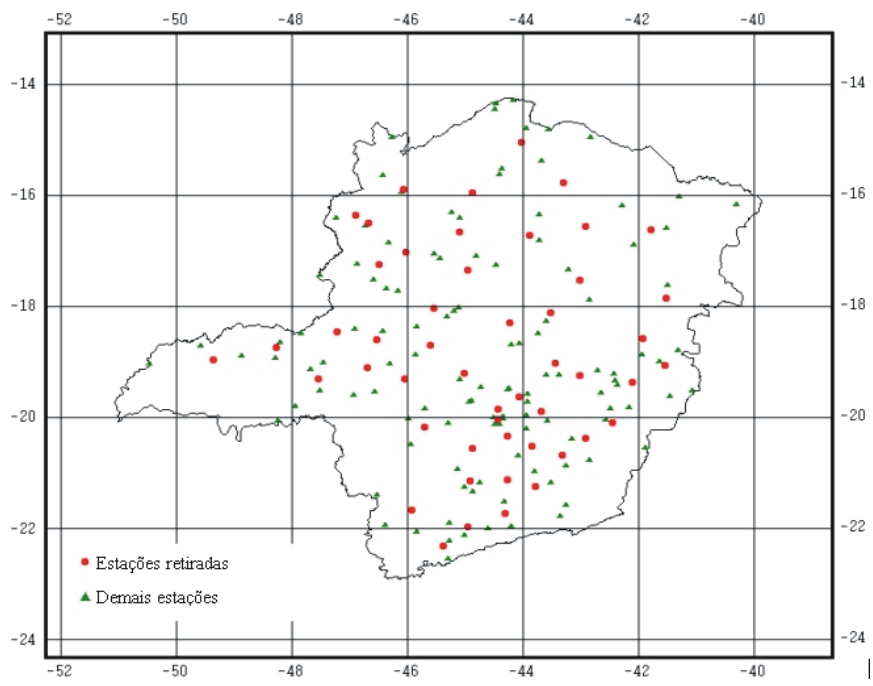

Figura 1. Localização das estações pluviográficas do Estado de Minas Gerais utilizadas no trabalho

Os valores interpolados dos parâmetros "K", "a", "b" e "c" foram armazenados a fim de que, posteriormente e para cada uma das 50 estações, fosse procedido o cálculo de $\mathrm{i}_{\mathrm{m}}$ (Eq. 1), utilizando-se todas as possíveis combinações entre os valores interpolados dos mesmos. Para tanto, considerou-se um período de retorno da precipitação (T) igual a 10 anos e a duração da precipitação (t) igual a $60 \mathrm{~min}$, com o que se obtiveram 625 diferentes valores de $\mathrm{i}_{\mathrm{m}}$ referentes a cada uma das combinações. Esses valores foram confrontados com o valor de $\mathrm{i}_{\mathrm{m}}$ calculado a partir dos valores reais de "K", "a", "b" e "c" da respectiva estação (determinados por Freitas et al., 
2001). Para cada combinação calculou-se, por intermédio da Eq. 3, o erro percentual entre o valor real de $\mathrm{i}_{\mathrm{m}}$ e o valor obtido a partir dos parâmetros interpolados.

$$
\mathrm{EP}=\frac{100\left(\mathrm{i}_{\mathrm{m} \mathrm{calc}}-\mathrm{i}_{\mathrm{m} \mathrm{int}}\right)}{i_{\mathrm{m} \mathrm{calc}}}
$$

em que:

$$
\begin{aligned}
& \text { EP - erro percentual entre os valores de } i_{m} \\
& i_{m \text { calc }}-i_{m} \text { calculado com os valores reais de "K", "a", "b", } \\
& i_{m \text { int }}-i_{m} \text { calculado com os valores interpolados de "K", } \\
& \text { "a", "b" e "c" }
\end{aligned}
$$

O valor de EP obtido pela Eq. 3 pode ser positivo ou negativo, que representa, respectivamente, sub ou superestimativa do valor de $i_{m}$.

Como a média dos valores de EP pouco significaria na determinação de um erro médio percentual (EM) proporcionado por cada combinação em cada estação, optou-se por calcular a média dos módulos de cada valor de EP para representar EM. Calculou-se, portanto, utilizando-se os valores do módulo de EP das 50 estações, o erro médio percentual (EM) de cada uma das 625 possíveis combinações entre os valores interpolados de "K", "a", "b" e "c".

\section{RESULTADOS E DISCUSSÃO}

$\mathrm{Na}$ Tabela 1 tem-se as combinações entre os valores interpolados que apresentaram os menores valores de erro médio percentual. Todos os valores de EM apresentados nesta tabela não diferem estatisticamente pelo teste t a nível de $10 \%$ de probabilidade, nela também são apresentados os valores das médias de EP (utilizadas para verificar se há tendência de sub ou superestimativa do valor de $\mathrm{i}_{\mathrm{m}}$ ), o mínimo e o máximo valor do módulo de EP, bem como a diferença entre ambos.

A média dos erros percentuais (média dos valores de EP) foi sempre negativa, mostrando tendência de todas as combinações apresentadas superestimarem a intensidade máxima média de precipitação calculada, o que significa trabalhar com uma margem de segurança adicional no projeto das obras de engenharia.

Percebe-se que, para 28 das 625 combinações realizadas, o erro médio percentual (EM) foi pequeno, com valores variando entre 18,65 e 19,83\% (valores estatisticamente não-diferentes). As 28 combinações listadas na Tabela 1 podem, portanto, ser recomendadas para a interpolação dos parâmetros da equação de chuvas intensas. Para essas 28 combinações, o erro percentual máximo variou entre 67,18 e 88,34\%, valores

\begin{tabular}{|c|c|c|c|c|c|c|c|c|c|}
\hline \multirow{2}{*}{ Combinação } & \multicolumn{4}{|c|}{ Forma de Interporlação } & $\mathrm{EP}_{\text {med }}{ }^{1}$ & $\mathrm{EM}^{2}$ & Min. $|\mathrm{EP}|^{3}$ & Máx. $|E P|^{4}$ & $\operatorname{Var}^{5}$ \\
\hline & K & $\mathrm{a}$ & $\mathrm{b}$ & $\mathrm{c}$ & \multicolumn{5}{|c|}{$(\%)$} \\
\hline 1 & ID5 & ID1 & ID5 & ID4 & $-11,28$ & 18,65 & 0,40 & 74,13 & 73,72 \\
\hline 2 & ID5 & ID1 & ID3 & ID5 & $-11,89$ & 18,77 & 1,11 & 67,71 & 66,60 \\
\hline 3 & ID5 & ID2 & ID3 & ID5 & $-11,98$ & 18,80 & 0,44 & 71,31 & 70,87 \\
\hline 4 & ID5 & ID2 & ID5 & ID4 & $-11,38$ & 18,86 & 0,52 & 73,73 & 73,21 \\
\hline 5 & ID5 & ID1 & ID4 & ID5 & $-12,46$ & 18,88 & 0,17 & 73,89 & 73,72 \\
\hline 6 & ID5 & ID1 & ID4 & ID4 & $-10,98$ & 18,92 & 1,23 & 75,18 & 73,95 \\
\hline 7 & ID5 & ID2 & ID4 & ID5 & $-12,55$ & 18,94 & 0,19 & 77,94 & 77,75 \\
\hline 8 & ID5 & ID3 & ID3 & ID5 & $-11,94$ & 18,95 & 0,16 & 75,70 & 75,54 \\
\hline 9 & ID5 & ID3 & ID4 & ID5 & $-12,52$ & 19,06 & 0,02 & 82,51 & 82,48 \\
\hline 10 & ID5 & ID3 & ID5 & ID4 & $-11,36$ & 19,10 & 0,29 & 75,54 & 75,25 \\
\hline 11 & ID5 & ID5 & ID4 & ID5 & $-12,38$ & 19,11 & 0,02 & 85,47 & 85,45 \\
\hline 12 & ID5 & ID4 & ID3 & ID5 & $-11,90$ & 19,12 & 0,48 & 78,14 & 77,66 \\
\hline 13 & ID5 & ID2 & ID4 & ID4 & $-11,08$ & 19,12 & 1,15 & 74,78 & 73,63 \\
\hline 14 & ID5 & ID4 & ID4 & ID5 & $-12,47$ & 19,14 & 0,21 & 85,04 & 84,84 \\
\hline 15 & ID5 & ID5 & ID3 & ID5 & $-11,82$ & 19,14 & 0,48 & 78,56 & 78,07 \\
\hline 16 & ID5 & ID1 & ID5 & ID5 & $-12,83$ & 19,19 & 0,28 & 76,58 & 76,30 \\
\hline 17 & ID5 & ID2 & ID5 & ID5 & $-12,93$ & 19,22 & 0,18 & 80,70 & 80,52 \\
\hline 18 & ID5 & ID4 & ID5 & ID4 & $-11,33$ & 19,25 & 0,63 & 77,98 & 77,35 \\
\hline 19 & ID5 & ID5 & ID5 & ID4 & $-11,25$ & 19,28 & 1,09 & 78,39 & 77,30 \\
\hline 20 & ID5 & ID1 & ID2 & ID5 & $-11,27$ & 19,32 & 0,63 & 67,57 & 66,94 \\
\hline 21 & ID5 & ID5 & ID5 & ID5 & $-12,75$ & 19,37 & 0,66 & 88,34 & 87,68 \\
\hline 22 & ID5 & ID3 & ID5 & ID5 & $-12,89$ & 19,38 & 0,20 & 85,33 & 85,13 \\
\hline 23 & ID5 & ID4 & ID5 & ID5 & $-12,84$ & 19,41 & 0,43 & 87,91 & 87,48 \\
\hline 24 & ID5 & ID2 & ID2 & ID5 & $-11,36$ & 19,47 & 0,40 & 67,18 & 66,78 \\
\hline 25 & ID5 & ID1 & ID3 & ID4 & $-10,50$ & 19,49 & 1,57 & 76,11 & 74,54 \\
\hline 26 & ID5 & ID3 & ID2 & ID5 & $-11,33$ & 19,68 & 0,40 & 67,48 & 67,07 \\
\hline 27 & ID5 & ID4 & ID2 & ID5 & $-11,29$ & 19,80 & 0,02 & 67,48 & 67,46 \\
\hline 28 & ID5 & ID5 & ID2 & ID5 & $-11,21$ & 19,83 & 0,48 & 67,48 & 67,00 \\
\hline
\end{tabular}
considerados aceitáveis em se tratando de intensidade máxima média de precipitação pluvial.

Tabela 1. Combinações entre parâmetros interpolados que apresentaram menor valor médio do módulo do erro percentual

\footnotetext{
Média dos erros percentuais

Mínimo valor do módulo de EP

${ }^{4}$ Máximo valor do módulo de EP

${ }^{5}$ Variação do erro percentual (diferença entre o máximo e o mínimo módulo de EP)
} 
De modo geral, percebe-se que a interpolação do parâmetro "K" foi melhor realizada utilizando-se o inverso da quinta potência da distância, em função de ser este um parâmetro cujo valor varia bastante (entre cerca de 500 e 11000). Assim, as estações mais próximas à localidade onde se deseja obter, por interpolação, o parâmetro "K", exerceram grande influência em seu valor, enquanto estações mais afastadas exerceram pouca influência.

O parâmetro "a" não apresentou grande sensibilidade ao expoente utilizado na interpolação via inverso da potência da distância, visto que tal parâmetro é o que possui menor magnitude e menor variação que os outros.

O parâmetro " $b$ " mostrou melhores respostas quando interpolado ao inverso de maiores potências da distância, sendo que o inverso da primeira potência não apresentou resultados satisfatórios em qualquer combinação.

O parâmetro "c" indicou melhores respostas quando interpolado utilizando-se o inverso da quinta potência da distância, apesar de também ter mostrado bons resultados para o inverso da quarta potência. Para este parâmetro, a interpolação utilizando-se o inverso das menores potências da distância, não apresentou resultados satisfatórios.

Percebe-se, na Tabela 1, que a combinação apresentada com o número 2 indicou menor variação entre o máximo e o mínimo valor do módulo do erro percentual (coluna Var da referida tabela) além de apresentar um dos menores valores máximos do módulo do erro percentual. Assim sendo, esta combinação, que toma " $\mathrm{K}$ " e "c" interpolados com o inverso da quinta potência da distância "a", interpolado com o inverso da distância e "b" interpolado com o inverso do cubo da distância, é a mais indicada para a realização de processos de interpolação dos parâmetros da equação de chuvas intensas.

\section{CONCLUSÕES}

Com base nos resultados obtidos, conclui-se que:

1. As combinações entre valores de "K", "a", "b", "c" interpolados utilizando-se o inverso das cinco diferentes potências da distância, apresentaram tendência de superestimativa do valor da intensidade máxima média de precipitação pluvial.

2. A interpolação de "K" e "c" com o inverso da quinta potência da distância, "a" com o inverso da distância e "b" com o inverso do cubo da distância, é recomendada por ser a combinação que apresentou menor variação entre o erro mínimo e o máximo, além de baixos valores de erro percentual máximo.

\section{LITERATURA CITADA}

Beltrame, L.F.S.; Lana, A.E.L.; Louzada, J.A.S. Chuvas intensas. Porto Alegre: IPH, UFRGS, 1991.69p.

Fendrich, R.; Freitas, C.O.A. Chuvas intensas no Estado do Paraná. Curitiba: PUC/Instituto de Saneamento Ambiental. 1992.45p.

Freitas, A.J.; Silva, D.D.; Pruski, F.F.; Pinto, F.A.; Pereira, S.B.; Gomes Filho, R.R.; Teixeira, A.F.; Baena, L.G.N.; Mello, L.T.A.; Novaes, L.F. Equações de chuvas intensas no Estado de Minas Gerais. Belo Horizonte: Companhia de Saneamento de Minas Gerais; Viçosa: Universidade Federal de Viçosa, 2001.65p.

Pinto, F.A.; Ferreira, P.A.; Pruski, F.F.; Alves, A.R.; Cecon, P.R. Equações de chuvas intensas para algumas localidades do Estado de Minas Gerais. Revista Engenharia Agrícola, Jaboticabal, v.16, n.1, p.91-104, 1996.

Pruski, F.F.; Calijuri, M.L.; Bhering, E.M.; Silva, J.M.A. Metodologia baseada no uso de sistemas de informações geográficas para obtenção de equações de chuvas intensas em qualquer localidade do Estado do Paraná. Revista Engenharia na Agricultura, Viçosa, v.5, n.3, p.254-265, 1997.

Pruski, F.F.; Silva, D.D.; Teixeira, A.F.; Silva, J.M.A.; Cecílio, R.A.; Silva, D.F. Plúvio 1.3: chuvas intensas para o Brasil. In: Congresso Brasileiro de Engenharia Agrícola, 31, 2002, Salvador. Anais... Salvador: Sociedade Brasileira de Engenharia Agrícola, 2002. CD-Rom

Silva, D.D.; Gomes Filho, R.R.; Pruski, F.F.; Pereira, S.B.; Novaes, L.F. Chuvas intensas no Estado da Bahia. Revista Brasileira de Engenharia Agrícola e Ambiental, Campina Grande, v.6, n.2, p.362-367, 2002.

Silva, D.D.; Pinto, F.R.L.; Pruski, F.F.; Pinto, F.A. Estimativa e espacialização dos parâmetros da equação de intensidadeduração-freqüência da precipitação para o Rio de Janeiro e o Espírito Santo. Revista Engenharia Agrícola, Jaboticabal, v.18, n.3, p.11-21, 1999a.

Silva, D.D.; Valverde, A.E.L.; Pruski, F.F.; Gonçalves, R.A.B. Estimativa e espacialização dos parâmetros da equação de intensidade-duração-freqüência da precipitação para o Estado de São Paulo. Revista Engenharia Agrícola, Jaboticabal, v.7, n.2, p.70-87, 1999b. 